\title{
On Some Equations on Non-Smooth Manifolds: Canonical Domains and Model Operators
}

\author{
Vladimir B. Vasilyev
}

\begin{abstract}
We describe different aspects of the theory of pseudo-differential equations on manifolds with non-smooth boundaries. Using a concept of special factorization for an elliptic symbol we consider distinct variants of this approach including asymptotic and discrete situations.
\end{abstract}

Key words: pseudo-differential equation, wave factorization, solvability, asymptotical expansion, discrete equation

\section{Introduction}

From 90s the author develops the theory of boundary value problems based on two principles [9]. These are a local principle and factorizability principle at a boundary point like $[2,15,3]$. The first principle was known earlier and it also was known as a freezing coefficients principle. Usually the second name corresponds to partial differential equations theory but the first name was introduced for multidimensional singular integral equations and more general for pseudo-differential equations. Main difference between differential and pseudo-differential operator is the following. A differential operator $\mathscr{D}$ has a local property i.e. if one takes two smooth functions $\varphi, \psi$ with non-intersecting supports and compose the operator $\psi \cdot \mathscr{D} \cdot \varphi$ then it leads to a zero operator. For a pseudo-differential operator $\mathscr{P}$ this property does not hold and we obtain for $\psi \cdot \mathscr{P} \cdot \varphi$ a compact operator only. This case permits to obtain rough properties for pseudo-differential equations and related boundary value problems namely Fredholm properties only in comparison with differential operators and boundary value problems where one has as a rule results on existence and uniqueness.

Vladimir B. Vasilyev

Chair of Differential Equations, Belgorod National Research University, Studencheskaya 14/1, Belgorod 308007, Russia, e-mail: vbv57@inbox.ru 
There are a lot of approaches to construct such a theory (see for example papers $[5,7,8])$. I have written many times $[11,17]$ what is difference between this consideration and others, it is choice of distinct key principles. In any case one needs to declare an invertibility of so-called local representatives of an initial pseudodifferential operator to describe its Fredholm properties.

Local principle and factorizability was first introduced in papers of I.B. Simonenko [15] (for multidimensional singular integral operators in Lebesgue $L_{p}$-spaces) and M.I. Vishik - G.I. Eskin [3] (for pseudo-differential operators in Sobolev - Slobodetskii $H^{s}$-spaces). For manifolds with a smooth boundary one uses an idea of "rectification of a boundary", and the problems reduces to a half-space case, for which a factorizability principle holds immediately because under localization at a boundary point and applying the Fourier transform we obtain well known onedimensional classical Riemann boundary value problem for upper and lower complex half-planes with a multidimensional parameter. This approach does not work if a boundary has at least one singular point like a conical point. One needs here other considerations and approaches.

\section{Domains and operators}

Our main goal is to describe possible solvability conditions for the pseudo-differential equation

$$
(A u)(x)=f(x), x \in D,
$$

where $D$ is manifold with a boundary, $A$ is pseudo-differential operator with the $\operatorname{symbol} A(x, \xi)$.

Such operators are defined locally by the formula

$$
u(x) \longmapsto \int_{\mathbf{R}^{m}} \int_{\mathbf{R}^{m}} A(x, \xi) \tilde{u}(y) e^{-i(x-y) \cdot \xi} d \xi d y
$$

if $D$ is a smooth compact manifold, because can use "freezing coefficients principle ", or in other words "local principle". For manifold with a smooth boundary we need new local formula for defining the operator $A$ : more precisely in inner points of $D$ we use the formula 1 , but in boundary points we need another formula

$$
u(x) \longmapsto \int_{\mathbf{R}_{+}^{m}} \int_{\mathbf{R}^{m}} A(x, \xi) u(y) e^{-i(x-y) \cdot \xi} d \xi d y .
$$

For invertibility of such operator (2) with symbol $A(\cdot, \xi)$ non-depending on spatial variable $x$ one can apply the theory of classical Riemann boundary problem for upper and lower complex half-planes with a parameter $\xi^{\prime}$. This step was systematically studied in the book [3]. But if the boundary $\partial D$ has at least one conical point, this approach is not effective. 
The conical point at the boundary is a such point, for which its neighborhood is diffeomorphic to the cone

$$
C_{+}^{a}=\left\{x \in \mathbf{R}^{m}: x_{m}>a\left|x^{\prime}\right|, x^{\prime}=\left(x_{1}, \ldots, x_{m-1}\right), a>0\right\},
$$

hence the local definition for pseudo-differential operator near the conical point is the following

$$
u(x) \longmapsto \int_{C_{+}^{a}} \int_{\mathbf{R}^{m}} A(x, \xi) u(y) e^{-i(x-y) \cdot \xi} d \xi d y .
$$

We consider the operator 1 in the Sobolev - Slobodetskii space $H^{s}\left(\mathbf{R}^{m}\right)$ with norm

$$
\|u\|_{s}^{2}=\int_{\mathbf{R}^{m}}|\tilde{u}(\xi)|^{2}(1+|\xi|)^{2 s} d \xi
$$

where $\tilde{u}(\xi)$ denotes the Fourier transform for $u$, and introduce the following class of symbols non-depending on spatial variable $x$ : $\exists c_{1}, c_{2}>0$, such that

$$
c_{1} \leq\left|A(\xi)(1+|\xi|)^{-\alpha}\right| \leq c_{2}, \xi \in \mathbf{R}^{m} .
$$

The number $\alpha \in \mathbf{R}$ we call the order of pseudo-differential operator $A$.

It is well-known that pseudo-differential operator with symbol $A(\xi)$ satisfying 3, is linear bounded operator acting from $H^{s}\left(\mathbf{R}^{m}\right)$ into $H^{s-\alpha}\left(\mathbf{R}^{m}\right)$ [3].

We are interested in studying invertibility operator 3 in corresponding Sobolev - Slobodetskii spaces. By definition, $H^{s}\left(C_{+}^{a}\right)$ consists of distributions from $H^{s}\left(\mathbf{R}^{m}\right)$ with support in $C_{+}^{a}$. The norm in the space $H^{s}\left(C_{+}^{a}\right)$ is induced by the norm $H^{s}\left(\mathbf{R}^{m}\right)$. We associate such operator with corresponding equation

$$
\left(A u_{+}\right)(x)=f(x), x \in C_{+}^{a},
$$

where right-hand side $f$ is chosen from the space $H_{0}^{s-\alpha}\left(C_{+}^{a}\right)$.

$H_{0}^{s}\left(C_{+}^{a}\right)$ is the space of distributions $S^{\prime}\left(C_{+}^{a}\right)$, which admit continuation on $H^{s}\left(\mathbf{R}^{m}\right)$. The norm in $H_{0}^{s}\left(C_{+}^{a}\right)$ is defined by

$$
\|f\|_{s}^{+}=\inf \|l f\|_{s}
$$

where infimum is chosen for all possible continuations $l$.

\section{Complex variables and wave factorization}

Below we will consider the symbols $A(\xi)$ satisfying the condition 4 .

Definition 1. Wave factorization of symbol $A(\xi)$ is called its representation in the form

$$
A(\xi)=A_{\neq}(\xi) A_{=}(\xi)
$$


where the factors $A_{\neq}(\xi), A_{=}(\xi)$ satisfy the following conditions:

1) $A_{\neq}(\xi), A_{=}(\xi)$ are defined everywhere without may be the points $\left\{\xi \in \mathbf{R}^{m}\right.$ : $\left.\left|\xi^{\prime}\right|^{2}=a^{2} \xi_{m}^{2}\right\}$;

2) $A_{\neq}(\xi), A_{=}(\xi)$ admit an analytical continuation into radial tube domains $T\left({ }^{*} C_{+}\right.$ ), $T\left({ }^{*}{ }_{-}^{a}\right)$ respectively, which satisfy the estimates

$$
\begin{gathered}
\left|A_{\neq}^{ \pm 1}(\xi+i \tau)\right| \leq c_{1}(1+|\xi|+|\tau|)^{ \pm \mathfrak{x}}, \\
\left|A_{=}^{ \pm 1}(\xi-i \tau)\right| \leq c_{2}(1+|\xi|+|\tau|)^{ \pm(\alpha-\mathfrak{x})}, \forall \tau \in C_{+}^{*} .
\end{gathered}
$$

The number $æ$ is called index of wave factorization.

Here $C_{+}^{*}$ is conjugate cone to $C_{+}^{a}$, and $\stackrel{*}{C_{-}^{a}}=-\stackrel{*}{C_{+}^{a}}$.

Example 1. Let

$$
A=-\frac{\partial^{2}}{\partial x_{1}^{2}}-\cdots-\frac{\partial^{2}}{\partial x_{m}^{2}}+k^{2}, \quad k \in \mathbf{R} \backslash\{\mathbf{0}\},
$$

and then according to some properties of the Fourier transform the symbol of this operator has the form

$$
A(\xi)=\xi_{1}^{2}+\xi_{2}^{2}+\cdots+\xi_{m}^{2}+k^{2} .
$$

The following equality is the wave factorization of the Helmholtz operator. We will write it as

$$
\begin{gathered}
\xi_{m}^{2}+\left|\xi^{\prime}\right|^{2}+k^{2}= \\
\left(\sqrt{a^{2}+1} \xi_{m}+\sqrt{a^{2} \xi_{m}^{2}-\left|\xi^{\prime}\right|^{2}-k^{2}}\right)\left(\sqrt{a^{2}+1} \xi_{m}-\sqrt{a^{2} \xi_{m}^{2}-\left|\xi^{\prime}\right|^{2}-k^{2}}\right)
\end{gathered}
$$

meaning for $\sqrt{a^{2} \xi_{m}^{2}-\left|\xi^{\prime}\right|^{2}-k^{2}}$ the boundary value

$$
\sqrt{a^{2}\left(\xi_{m}+i 0\right)^{2}-\left|\xi^{\prime}\right|^{2}-k^{2}}
$$

\section{Pseudo-differential equations and solvability}

To describe a solvability picture for a model elliptic pseudo differential equation (5) in 2-dimensional cone $C_{+}^{a}=\left\{x \in \mathbf{R}^{2}: x_{2}>a\left|x_{1}\right|, a>0\right\}$ the author earlier considered a special singular integral operator [9]

$$
\left(K_{a} u\right)(x)=\frac{a}{2 \pi^{2}} \lim _{\tau \rightarrow 0+} \int_{\mathbf{R}^{2}} \frac{u(y) d y}{\left(x_{1}-y_{1}\right)^{2}-a^{2}\left(x_{2}-y_{2}+i \tau\right)^{2}} .
$$


This operator served a conical singularity in the general theory of boundary value problems for elliptic pseudo differential equations on manifolds with a non-smooth boundary. The operator $K_{a}$ is a convolution operator, and the parameter $a$ is a size of an angle, $x_{2}>a\left|x_{1}\right|, a=\cot \alpha$.

One of author's main result [9] is the following (we formulate it for $m=2$ for simplicity)

Theorem 1. If elliptic symbol $A(\xi)$ admits wave factorization with respect to the cone $C_{+}^{a}$ and $|\mathfrak{x}-s|<1 / 2$ then the equation (5) has a unique solution

$$
\widetilde{u}(\xi)=A_{\neq}^{-1}(\xi)\left(K_{a} \widetilde{l v}\right)(\xi)
$$

where $l v$ is an arbitrary continuation of $v$ on the whole $H^{s}\left(\mathbf{R}^{2}\right)$.

A priori estimate holds

$$
\left\|u_{+}\right\|_{s} \leq c\|f\|_{s-\alpha}^{+}
$$

Below we will mention other possible situations.

\subsection{Boundary value problems}

If $\mathfrak{x}-s=n+\delta, n \in \mathbf{N},|\delta|<1 / 2$, then one has the formula for a general solution of the equation (5), and this formula contains a certain number of arbitrary functions from corresponding Sobolev-Slobodetskii spaces [9]. To obtain the uniqueness theorem one needs to add some complementary conditions as a rule these are boundary conditions.

Some classical variants are considered in [9], some new constructions are described in [17].

\subsection{Equations with potentials}

It is possible that $\mathfrak{x}-s=n+\delta,-n \in \mathbf{N},|\delta|<1 / 2$, then the equation (5) is overdetermined so that one needs to add some unknowns. According to the special representation for a solution of the equation (5) these unknowns should have a potential like form [9].

\section{Asymptotical variants}

For $|\mathfrak{x}-s|<1 / 2$ one has the existence and uniqueness theorem [9]

$$
\widetilde{u}(\xi)=A_{\neq}^{-1}(\xi)\left(K_{a} \widetilde{l v}\right)(\xi)
$$


where $l v$ is an arbitrary continuation of $v$ on the whole $H^{s}\left(\mathbf{R}^{2}\right)$.

\subsection{Preliminaries}

The formula (6) can be treated as a convolution of the distribution

$$
K_{a}(\xi)=\frac{a}{2 \pi^{2}} \frac{1}{\xi_{1}^{2}-a^{2} \xi_{2}^{2}}
$$

with a basic function $u(\xi)$. If so it is interesting to study behavior of the operator (6) for limit cases $(a=0, a=+\infty)$ from convolution point of view.

Let $S\left(\mathbf{R}^{2}\right)$ be the Schwartz space of infinitely differentiable rapidly decreasing at infinity functions then $S^{\prime}\left(\mathbf{R}^{2}\right)$ is a corresponding space of distributions over $S\left(\mathbf{R}^{2}\right)$.

When $a \rightarrow+\infty$ one obtains [10] the following limit distribution

$$
\lim _{a \rightarrow \infty} \frac{a}{2 \pi^{2}} \frac{1}{\xi_{1}^{2}-a^{2} \xi_{2}^{2}}=\frac{i}{2 \pi} \mathscr{P} \frac{1}{\xi_{1}} \otimes \delta\left(\xi_{2}\right),
$$

where the notation for distribution $\mathscr{P}$ is taken from V.S. Vladimirov's books $[18,19]$, and $\otimes$ denotes the direct product of distributions. Here $\delta$ denotes onedimensional Dirac mass-function which acts on $\varphi \in S(\mathbf{R})$ in the following way

$$
(\delta, \varphi)=\varphi(0)
$$

and the distribution $\mathscr{P} \frac{1}{x}$ is defined by the formula

$$
\left(\mathscr{P} \frac{1}{x}, \varphi\right)=v \cdot p \cdot \int_{-\infty}^{+\infty} \frac{\varphi(x) d x}{x} \equiv \lim _{\varepsilon \rightarrow 0+}\left(\int_{-\infty}^{-\varepsilon}+\int_{\varepsilon}^{+\infty}\right) \frac{\varphi(x) d x}{x} .
$$

Our main goal in this paper is obtaining an asymptotical expansion for the twodimensional distribution

$$
K_{a}\left(\xi_{1}, \xi_{2}\right) \equiv \frac{a}{2 \pi^{2}} \frac{1}{\xi_{1}^{2}-a^{2} \xi_{2}^{2}}
$$

with respect to small $a^{-1}$. It is defined by the corresponding formula $\forall \varphi \in S\left(\mathbf{R}^{2}\right)$

$$
\left(K_{a}, \varphi\right)=\frac{a}{2 \pi^{2}} \int_{\mathbf{R}^{2}} \frac{\varphi\left(\xi_{1}, \xi_{2}\right) d \xi}{\xi_{1}^{2}-a^{2} \xi_{2}^{2}} .
$$




\subsection{Asymptotical representation for a solution}

Below we denote $l v \equiv V$.

Theorem 2. If the symbol $A(\xi)$ admits a wave factorization with respect to the cone $C_{+}^{a}$ and $|\mathfrak{x}-s|<1 / 2$ then the equation 1 has a unique solution in the space $H^{s}\left(C_{+}^{a}\right)$, and for a large a it can be represented in the form

$$
\begin{gathered}
\widetilde{u}(\xi)=\frac{i}{2 \pi} A_{\neq}^{-1}(\xi) v \cdot p \cdot \int_{-\infty}^{+\infty} \frac{\left(A_{=}^{-1} \widetilde{V}\right)\left(\eta_{1}, \xi_{2}\right) d \eta_{1}}{\xi_{1}-\eta_{1}}+ \\
A_{\neq}^{-1}(\xi) \sum_{m, n} c_{m, n}(a) \int_{-\infty}^{+\infty}\left(\xi_{1}-\eta_{1}\right)^{m}\left(A_{=}^{-1} \widetilde{V}\right)_{\xi_{2}}^{(n)}\left(\eta_{1}, \xi_{2}\right) d \eta_{1}
\end{gathered}
$$

assuming $\widetilde{V} \in S\left(\mathbf{R}^{2}\right), A_{=}^{-1} \widetilde{V}$ means the function $A_{=}^{-1}(\xi) \widetilde{V}(\xi)$.

Proof. Let $\varphi \in S\left(\mathbf{R}^{2}\right)$.

A formal using the Maclaurin formula for the first integral in 4 will lead to the following result

$$
\left(K_{a}, \varphi\right)=\frac{1}{2 \pi^{2}} \sum_{k=0}^{\infty} \frac{b^{k}}{k !} \int_{-N}^{+N} \varphi_{\xi_{2}}^{(k)}\left(\xi_{1}, 0\right)\left(\int_{-N}^{+N} \frac{t^{k} d t}{\xi_{1}^{2}-t^{2}}\right) d \xi_{1}
$$

and we need to give a certain sense for the expression in brackets.

Let us denote

$$
T_{k, N}\left(\xi_{1}\right) \equiv \int_{-N}^{+N} \frac{t^{k} d t}{\xi_{1}^{2}-t^{2}}
$$

and reproduce some calculations.

First $T_{k, N}\left(\xi_{1}\right) \equiv 0, \forall k=2 n-1, n \in \mathbf{N}$. So the non-trivial case is $k=2 n, n \in \mathbf{N}$. Let us remind $T_{0, \infty}\left(\xi_{1}\right)=\pi i 2^{-1} \xi_{1}^{-1}[10,11]$. For other cases we can calculate this integral. we have the following

$$
k=2 \text {, }
$$

$$
T_{2, N}\left(\xi_{1}\right)=-2 N-2^{-1} \xi_{1}^{-1} \ln \frac{N-\xi_{1}}{N+\xi_{1}}+\pi i 2^{-1} \xi_{1}^{-1}
$$

$k=4$,

$$
T_{4, N}\left(\xi_{1}\right)=-2 / 3 N^{3}-2 \xi_{1}^{2} N-2^{-1} \xi_{1}^{3} \ln \frac{N-\xi_{1}}{N+\xi_{1}}+\pi i 2^{-1} \xi_{1}^{3}
$$

$k=6$,

$$
T_{6, N}\left(\xi_{1}\right)=-2 / 5 N^{5}-2 / 3 \xi_{1}^{2} N^{3}-2 \xi_{1}^{5} N-2^{-1} \xi_{1}^{5} \ln \frac{N-\xi_{1}}{N+\xi_{1}}+\pi i 2^{-1} \xi_{1}^{5},
$$


and so on. One can easily write all expressions for arbitrary $T_{2 n, N}\left(\xi_{1}\right)$.

In general one can write

$$
T_{2 n, N}\left(\xi_{1}\right)=P_{2 n-1}\left(N, \xi_{1}\right)-2^{-1} \xi_{1}^{2 n-1} \ln \frac{N-\xi_{1}}{N+\xi_{1}}+\pi i 2^{-1} \xi_{1}^{2 n-1}
$$

where $P_{2 n-1}\left(N, \xi_{1}\right)$ is a certain polynomial of order $2 n-1$ on variables $N, \xi_{1}$.

Therefore instead of the formula (10) we can write

$$
\left(K_{a}, \varphi\right)=\frac{i}{2 \pi}\left(\mathscr{P} \frac{1}{\xi_{1}} \otimes \delta\left(\xi_{2}\right), \varphi\right)+
$$

$\frac{1}{2 \pi^{2}} \sum_{n=1}^{\infty} \frac{b^{2 n}}{(2 n) !} \int_{-N}^{+N} \varphi_{\xi_{2}}^{(2 n)}\left(\xi_{1}, 0\right)\left(P_{2 n-1}\left(N, \xi_{1}\right)-2^{-1} \xi_{1}^{2 n-1} \ln \frac{N-\xi_{1}}{N+\xi_{1}}+\pi i 2^{-1} \xi_{1}^{2 n-1}\right) d \xi_{1}$.

Let us describe the polynomial $P_{2 n-1}\left(N, \xi_{1}\right)$ more precisely. Obviously

$$
P_{2 n-1}\left(N, \xi_{1}\right)=c_{2 n-1} N^{2 n-1}+c_{2 n-3} N^{2 n-3} \xi_{1}^{2}+\cdots+c_{1} N \xi_{1}^{2 n-1} .
$$

Further we rewrite the equality (11) in the following form

$$
\begin{gathered}
\left(K_{a}, \varphi\right)=\frac{i}{2 \pi}\left(\mathscr{P} \frac{1}{\xi_{1}} \otimes \delta\left(\xi_{2}\right), \varphi\right)+ \\
\frac{1}{2 \pi^{2}} \sum_{n=1}^{\infty} \frac{b^{2 n}}{(2 n) !} \sum_{k=1}^{n} c_{2 k-1} N^{2 k-1} \int_{-N}^{+N} \varphi_{\xi_{2}}^{(2 n)}\left(\xi_{1}, 0\right) \xi_{1}^{2 k-1} d \xi_{1}- \\
\frac{1}{4 \pi^{2}} \sum_{n=1}^{\infty} \frac{b^{2 n}}{(2 n) !} \int_{-N}^{+N} \varphi_{\xi_{2}}^{(2 n)}\left(\xi_{1}, 0\right) \xi_{1}^{2 n-1} \ln \frac{N-\xi_{1}}{N+\xi_{1}} d \xi_{1}+\frac{i}{4 \pi} \sum_{n=1}^{\infty} \frac{b^{2 n}}{(2 n) !} \int_{-N}^{+N} \varphi_{\xi_{2}}^{(2 n)}\left(\xi_{1}, 0\right) \xi_{1}^{2 n-1} d \xi_{1}
\end{gathered}
$$

We will start from two last summands. The second summand does not play any role because

$$
\lim _{N \rightarrow+\infty} \ln \frac{N-\xi_{1}}{N+\xi_{1}}=0 .
$$

The third summand we will represent according to lemma 1 (see below) taking into account that we can pass to the limit under $N \rightarrow+\infty$

$$
\frac{i}{4 \pi} \sum_{n=1}^{\infty} \frac{b^{2 n}}{(2 n) !}\left(\widetilde{\delta^{(2 n-1)}}\left(\xi_{1}\right) \otimes \delta^{(2 n)}\left(\xi_{2}\right), \varphi\right)
$$

For the first summand we consider separately the case $N b \sim 1(N \rightarrow \infty, b \rightarrow 0)$. in other words we consider a special limit to justify the decomposition. Then 


$$
\begin{aligned}
& \frac{1}{2 \pi^{2}} \sum_{n=1}^{\infty} \frac{b^{2 n}}{(2 n) !} \sum_{k=1}^{n} c_{2 k-1} N^{2 k-1} \int_{-N}^{+N} \varphi_{\xi_{2}}^{(2 n)}\left(\xi_{1}, 0\right) \xi_{1}^{2 k-1} d \xi_{1} \sim \\
& \frac{1}{2 \pi^{2}} \sum_{n=1}^{\infty} \frac{1}{(2 n) !} \sum_{k=1}^{n} c_{2 k-1} b^{2 n-2 k+1} \int_{-\infty}^{+\infty} \varphi_{\xi_{2}}^{(2 n)}\left(\xi_{1}, 0\right) \xi_{1}^{2 k-1} d \xi_{1} .
\end{aligned}
$$

Therefore

$$
\begin{aligned}
& \frac{1}{2 \pi^{2}} \sum_{n=1}^{\infty} \frac{1}{(2 n) !} \sum_{k=1}^{n} c_{2 k-1} b^{2 n-2 k+1} \int_{-\infty}^{+\infty} \varphi_{\xi_{2}}^{(2 n)}\left(\xi_{1}, 0\right) \xi_{1}^{2 k-1} d \xi_{1}= \\
& \frac{1}{2 \pi^{2}} \sum_{n=1}^{\infty} \frac{1}{(2 n) !} \sum_{k=1}^{n} c_{2 k-1} b^{2 n-2 k+1}\left(\widetilde{\left.\delta^{2 k-1}\right)}\left(\xi_{1}\right) \otimes \delta^{(2 n)}\left(\xi_{2}\right), \varphi\right)
\end{aligned}
$$

One can note if desirable

$$
c_{2 k-1}=-2\left(1+\frac{1}{3}+\cdots+\frac{1}{2 k-1}\right) .
$$

Further details one can find in [13]. $\triangle$

\section{Discrete variants}

\subsection{Discrete functions and operators: preliminaries and examples}

Given function $u_{d}$ of a discrete variable $\tilde{x} \in \mathbf{Z}^{m}$ we define its discrete Fourier transform by the series

$$
\left(F_{d} u_{d}\right)(\xi) \equiv \widetilde{u}_{d}(\xi)=\sum_{\tilde{x} \in \mathbf{Z}^{m}} e^{i \tilde{x} \cdot \xi} u_{d}(\tilde{x}), \quad \xi \in \mathbf{T}^{m}
$$

where $\mathbf{T}^{m}=[-\pi, \pi]^{m}$ and partial sums are taken over cubes

$$
Q_{N}=\left\{\tilde{x} \in \mathbf{Z}^{m}: \tilde{x}=\left(\tilde{x}_{1}, \cdots, \tilde{x}_{m}\right), \max _{1 \leq k \leq m}\left|\tilde{x}_{k}\right| \leq N\right\}
$$

One can define some discrete operators for such functions $u_{d}$.

Example 2. If $K(x), x \in \mathbf{R}^{m} \backslash\{0\}$, is a Calderon-Zygmund kernel, then the corresponding operator is defined by the formula

$$
\left(K_{d} u_{d}\right)(\tilde{x})=\sum_{\tilde{y} \in \mathbf{Z}^{m}, \tilde{y} \neq \tilde{x}} K(\tilde{x}-\tilde{y}) u_{d}(\tilde{y}), \quad \tilde{x} \in \mathbf{Z}^{m} .
$$

Example 3. If a first order finite difference of a discrete variable $\tilde{x}_{k}$ is defined by 


$$
\delta_{k} u_{d}(\tilde{x})=u_{d}\left(\tilde{x}_{k}+1\right)-u_{d}\left(\tilde{x}_{k}\right),
$$

then the discrete Laplacian is

$$
\left(\Delta_{d} u_{d}\right)(\tilde{x})=\sum_{k=1}^{m}\left(u_{d}\left(\tilde{x}_{k}+2\right)-2 u_{d}\left(\tilde{x}_{k}+1\right)+u_{d}\left(\tilde{x}_{k}\right)\right),
$$

and its discrete Fourier transform is the function

$$
\left(F_{d} \Delta_{d} u_{d}\right)(\xi)=\sum_{k=1}^{m}\left(e^{i \xi_{k}}-1\right)^{2} .
$$

Let $D \subset \mathbf{R}^{m}$ be a sharp convex cone, $D_{d} \equiv D \cap \mathbf{Z}^{m}$, and $L_{2}\left(D_{d}\right)$ be a space of functions of discrete variable defined on $D_{d}$, and $A(\tilde{x})$ be a given function of a discrete variable $\tilde{x} \in \mathbf{Z}^{m}$. We consider the following types of operators

$$
\left(A_{d} u_{d}\right)(\tilde{x})=\int_{\mathbf{T}^{m}} \sum_{\tilde{y} \in D_{d}} e^{i(\tilde{y}-\tilde{x}) \cdot \xi} \widetilde{A}_{d}(\xi) \tilde{u}_{d}(\xi) d \xi, \quad \tilde{x} \in D_{d}
$$

and introduce the function

$$
\widetilde{A}_{d}(\xi)=\sum_{\tilde{x} \in \mathbf{Z}^{m}} e^{i \tilde{x} \cdot \xi} A(\tilde{x}), \quad \xi \in \mathbf{T}^{m} .
$$

Definition 2. The function $\widetilde{A}_{d}(\xi)$ is called a symbol of the operator $A_{d}$, and this symbol is called an elliptic symbol if $\tilde{A}_{d}(\xi) \neq 0, \forall \xi \in \mathbf{T}^{m}$.

Remark 1. If $D=\mathbf{R}^{m}$ then an ellipticity is necessary and sufficient condition for the operator $A_{d}$ to be invertible in the space $L_{2}\left(\mathbf{Z}^{m}\right)$.

Remark 2. One can define a general pseudo-differential operator with symbol $\tilde{A}(\tilde{x}, \xi)$ depending on a spatial discrete variable $\tilde{x}$ by the similar formula

$$
\left(A_{d} u_{d}\right)(\tilde{x})=\int_{\mathbf{T}^{m}} \sum_{\tilde{y} \in D_{d}} e^{i(\tilde{y}-\tilde{x}) \cdot \xi} \widetilde{A}(\tilde{x}, \xi) \tilde{u}_{d}(\xi) d \xi, \quad \tilde{x} \in D_{d}
$$

but taking into account a local principle [2] the main aim in this situation is describing invertibility conditions for model operators like (12) in canonical domains $D_{d}$.

Below we will refine the lattice $\mathbf{Z}^{m}$ and introduce more convenient space scale.

\subsection{Discrete Sobolev-Slobodetskii spaces}

We consider here refined lattice $h \mathbf{Z}^{m}, h>0$, and define corresponding discrete Fourier transform. If a function of a discrete variable is defined on a lattice $h \mathbf{Z}^{m}$ then its discrete Fourier transform can be introduced by the formula 


$$
\left(\tilde{u}_{d}\right)(\xi)=\sum_{\tilde{x} \in h \mathbf{Z}^{m}} u_{d}(\tilde{x}) e^{i \tilde{x} \cdot \xi} h^{m}, \quad \xi \in \hbar \mathbf{T}^{m},
$$

where $\hbar=h^{-1}$.

Let $H^{s}\left(h \mathbf{Z}^{m}\right)$ denotes the space of functions of a discrete variable for which

$$
\left\|u_{d}\right\|_{s}^{2} \equiv \int_{\hbar \mathbf{T}^{m}}\left|\widetilde{u}_{d}(\xi)\right|^{2}\left(1+\left|\sigma_{\Delta_{d}}(h)(\xi)\right|\right)^{s} d \xi<+\infty,
$$

where

$$
\sigma_{\Delta_{d}}(h)(\xi)=h^{-2} \sum_{k=1}^{m}\left(e^{i h \xi_{k}}-1\right)^{2}, \quad \xi \in \hbar \mathbf{T}^{m}
$$

\subsection{Solvability for discrete equations}

\subsubsection{Conical case and periodic Bochner kernel}

Let $D$ be a sharp convex cone, and $\stackrel{*}{D}$ be a conjugate cone for $D$, i.e.,

$$
\stackrel{*}{D}=\left\{x \in \mathbf{R}^{m}: x \cdot y>0, y \in D\right\} .
$$

Let $T(\stackrel{*}{D}) \subset \mathbf{C}^{m}$ be a set of the type $\mathbf{T}^{m}+i \stackrel{*}{D}$. For $\mathbf{T}^{m} \equiv \mathbf{R}^{m}$ such a domain of multidimensional complex space is called a radial tube domain over the cone $\stackrel{*}{D}$ $([1,18,19])$. We introduce the function

$$
B_{d}(z)=\sum_{\tilde{x} \in D_{d}} e^{i \tilde{x} \cdot z}, \quad z=\xi+i \tau, \quad \xi \in \mathbf{T}^{m}, \quad \tau \in \stackrel{*}{D}
$$

and define the operator

$$
\left(B_{d} u\right)(\xi)=\lim _{\tau \rightarrow 0} \int_{\mathbf{T}^{m}} B_{d}(z-\eta) u_{d}(\eta) d \eta .
$$

Lemma 1. For arbitrary $u_{d} \in L_{2}\left(\mathbf{Z}^{m}\right)$, the following property

$$
F_{d} P_{D_{d}} u_{d}=B_{d} F_{d} u_{d}
$$

holds.

Let us define the subspace $A\left(\mathbf{T}^{m}\right) \subset L_{2}\left(\mathbf{T}^{m}\right)$ consisting of functions which admit a holomorphic continuation into $T(\stackrel{*}{D})$ and satisfy the condition

$$
\sup _{\substack{\tau \in D \\ \tau}} \int_{\mathbf{T}^{m}}\left|\tilde{u}_{d}(\xi+i \tau)\right|^{2} d \xi<+\infty .
$$


In other words, the space $A\left(\mathbf{T}^{m}\right) \subset L_{2}\left(\mathbf{T}^{m}\right)$ consists of boundary values of holomorphic in $T(\stackrel{*}{D})$ functions.

Let us denote

$$
B\left(\mathbf{T}^{m}\right)=L_{2}\left(\mathbf{T}^{m}\right) \ominus A\left(\mathbf{T}^{m}\right),
$$

so that $B\left(\mathbf{T}^{m}\right)$ is a direct complement of $A\left(\mathbf{T}^{m}\right)$ in $L_{2}\left(\mathbf{T}^{m}\right)$.

\subsubsection{A jump problem}

We formulate the problem in the following way: finding a pair of functions $\Phi^{ \pm}, \Phi^{+} \in$ $A\left(\mathbf{T}^{m}\right), \Phi^{-} \in B\left(\mathbf{T}^{m}\right)$, such that

$$
\Phi^{+}(\xi)-\Phi^{-}(\xi)=g(\xi), \quad \xi \in \mathbf{T}^{m}
$$

where $g(\xi) \in L_{2}\left(\mathbf{T}^{m}\right)$ is given.

Lemma 2. The operator $B_{d}: L_{2}\left(\mathbf{T}^{m}\right) \rightarrow A\left(\mathbf{T}^{m}\right)$ is a bounded projector. A function $u_{d} \in L_{2}\left(D_{d}\right)$ iff its Fourier transform $\tilde{u}_{d} \in A\left(\mathbf{T}^{m}\right)$.

Theorem 3. The jump problem has unique solution for arbitrary right-hand side from $L_{2}\left(\mathbf{T}^{m}\right)$.

Example 4. If $m=2$ and $D$ is the first quadrant in a plane then a solution of a jump problem is given by formulas

$$
\begin{gathered}
\Phi^{+}(\xi)=\frac{1}{(4 \pi i)^{2}} \lim _{\tau \rightarrow 0} \int_{-\pi}^{\pi} \int_{-\pi}^{\pi} \cot \frac{\xi_{1}+i \tau_{1}-t_{1}}{2} \cot \frac{\xi_{2}+i \tau_{2}-t_{2}}{2} g\left(t_{1}, t_{2}\right) d t_{1} d t_{2} \\
\Phi^{-}(\xi)=\Phi^{+}(\xi)-g(\xi), \quad \tau=\left(\tau_{1}, \tau_{2}\right) \in D .
\end{gathered}
$$

\subsubsection{A general statement}

It looks as follows. Finding a pair of functions $\Phi^{ \pm}, \Phi^{+} \in A\left(\mathbf{T}^{m}\right), \Phi^{-} \in B\left(\mathbf{T}^{m}\right)$, such that

$$
\Phi^{+}(\xi)=G(\xi) \Phi^{-}(\xi)+g(\xi), \quad \xi \in \mathbf{T}^{m},
$$

where $G(\xi), g(\xi)$ are given periodic functions. If $G(\xi) \equiv 1$, we have the jump problem (3).

Like classical studies $[4,6]$, we want to use a special representation for an elliptic symbol to solve the problem (4). 


\subsubsection{Periodic wave factorization}

Let us denote $H^{s}\left(D_{d}\right)$ a subspace of $H^{s}\left(\mathbf{Z}^{m}\right)$ consisting of functions of discrete variable $\tilde{x}$ for which their supports belong to $\overline{D_{d}}$, and $\widetilde{H}^{s}\left(D_{d}\right), \widetilde{H}^{s}\left(\mathbf{Z}^{m}\right)$ their Fourier images.

Lemma 3. For $|s|<1 / 2$, the operator $B_{d}$ is a bounded projector $\widetilde{H}^{s}\left(\mathbf{Z}^{m}\right) \rightarrow$ $\widetilde{H}^{s}\left(D_{d}\right)$, and a jump problem has unique solution $\Phi^{+} \in \widetilde{H}^{s}\left(D_{d}\right), \Phi^{-} \in \widetilde{H}^{s}\left(\mathbf{Z}^{m} \backslash D_{d}\right)$ for arbitrary $g \in \widetilde{H}^{s}\left(\mathbf{Z}^{m}\right)$.

Definition 3. Periodic wave factorization for elliptic symbol $\tilde{A}(\xi)$ is called its representation in the form

$$
\tilde{A}_{d}(\xi)=\tilde{A}_{\neq}(\xi) \tilde{A}_{=}(\xi)
$$

where the factors $A_{\neq}^{ \pm 1}(\xi), A_{=}^{ \pm 1}(\xi)$ admit bounded holomorphic continuation into domains $T( \pm \stackrel{*}{D})$.

Theorem 4. If $|s|<1 / 2$ and the elliptic symbol $\tilde{A}_{d}(\xi) \in S_{\alpha}\left(\mathbf{T}^{m}\right)$ admits periodic wave factorization, then the operator $A_{d}$ is invertible in the space $H^{s}\left(D_{d}\right)$.

\section{Conclusion}

As it was shown all aspects of this problem of solving the equation (5) are closely related and use similar ideas and methods. Author hopes that in future it will be possible to unit these considerations in a general theory of elliptic pseudo-differential equations on manifolds with non-smooth boundaries.

\section{References}

1. S. Bochner, W.T. Martin, Functions of several complex variables. Moscow, Publ. Foreign Lit., 1951 (Russian).

2. Milkhin S.G., Prößdorf S. Singular integral operators. Berlin, Akademie-Verlag, 1986.

3. Eskin G.I. Boundary value problems for elliptic pseudodifferential equations. AMS, Providence, 1981.

4. F.D. Gakhov, Boundary problems. Moscow, Nauka, 1977. (Russian)

5. R.B. Melrose, Pseudodifferential operators, corners and singular limits. In: Proceeding of the International Congress of Mathematicians, 21-29 August 1990, Kyoto, Japan, I. Satake (ed.), Springer-Verlag, Tokyo-Berlin-Heidelberg-New York (1991), V. I, pp. 217-234.

6. N.I. Muskhelishvili, Singular integral equations. Moscow, Nauka, 1968. (Russian)

7. S.A. Nazarov, B.A. Plamenevsky, Elliptic problems in domains with piecewise smooth boundaries., Walter de Gruyter, 1994.

8. B.-W. Schulze, Pseudo-differential boundary value problems, conical singularities, and asymptotics. Berlin, 1994.

9. V. B. Vasil'ev, Wave factorization of elliptic symbols: theory and applications. Introduction to the boundary value problems in non-smooth domains. Kluwer Academic Publishers, 2000. 
10. V.B. Vasilyev, Asymptotical analysis of singularities for pseudo differential equations in canonical non-smooth domains. Integral Methods in Science and Engineering. Computational and Analytic Aspects. Eds. C.Constanda, P.J. Harris. Birkhäuser, Boston, 2011, 379-390.

11. V.B. Vasilyev, Pseudo differential equations on manifolds with non-smooth boundaries. In: Differential and Difference Equations and Applications. Springer Proc. Math. \& Stat. 47 (2013). P.625-637.

12. A.V. Vasilyev, V.B. Vasilyev, Discrete singular integrals in a half-space. In: Current Trends in Analysis and its Applications. Research Perspectives. Birkhäuser, Basel, 2015. P. 663-670.

13. A.V. Vasilyev, On asymptotical expansions for certain singular integrals: 2-dimensional case. ArXiv: 1512.08237. P. 1-9.

14. Vasilyev V.B. Potentials for elliptic boundary value problems in cones. Siberian Electron. Math. Repts. 13 (2016). P. 1129-1149. (Russian)

15. I.B. Simonenko, Local method in the theory of translation invariant operators and their envelopings. Rostov on Don, 2007. (Russian)

16. Vasilyev V.B. Multipliers of Fourier integrals, pseudodifferential equations, the wave factorization, boundary value problems. Editorial URSS, Moscow, 2nd edition, 2010. (Russian)

17. V. B. Vasilyev, Fourier multipliers, pseudo differential equations, wave factorization, boundary value problems. 2nd Edition, Moscow, 2010. (Russian)

18. V.S. Vladimirov, Methods of functions theory of several complex variables. Moscow, 1964 (Russian)

19. V.S. Vladimirov, Distributions in mathematical physics. Moscow, 1979. (Russian) 